\title{
Vortex guide under a Lorentz force.
}

\author{
C. A. Aguirre ${ }^{1}$, Q. Martins ${ }^{2}$, José José Barba-Ortega ${ }^{3}$ \\ ${ }^{1,2}$ Universidade Federal de Mato-Grosso, Cuiabá - Brasil \\ ${ }^{3}$ Universidad Nacional de Colombia, Bogota - Colombia
}

Recibido: 04 de octubre de 2019.

Aprobado: 06 de diciembre de 2019.

\begin{abstract}
In the present work we study the effect that the metallic quality of the contacts by which an external current is applied, has on the magnetic properties of a superconducting sample. We analyze the magnetization, magnetic susceptibility, Cooper pair density as applied magnetic field function. The contacts are simulated using the $\gamma$ parameter, and anisotropy is present in sections with different critical temperatures modeled by the $\tau(x, y)$ function in the Ginzburg-Landau formalism. We establish how the nature of the contacts and the presence of the weak Lorentz force influence the magnetic response and the vortex state of the sample.
\end{abstract}

Keywords: Magnetization, Mesoscopics, Ginzburg Landau, Superconductor. 


\section{INTRODUCTION}

Many have been the advances during the last years in the area of superconductivity, among these, there are the theoretical and experimental development of superconductors of one, two and three bands, including the novel fractional vortices, this added to systems that include inclusion of external currents, applications to small variations of magnetic fields (Squids), topological superconductors and exotic phases that present these types of systems, such as Hopfions, Skirmions and Kinks among others [1-8]. This vast interest has been developed given the special properties that these systems have in superconducting state, for example in the case of type I superconductors, the conduction of electrical currents without loss Ohmincas, shielding of external fields and anchoring of materials, through pinnings and anti-pinnings and for type II superconductors, coordinated movement of superconductive state losses (vortices), oscillations in heat capacity and magnetic susceptibility even performing studies of vortex / anti-vortice annihilation, generating power losses in the system. Given this, metastable characteristics of different types of compounds have been studied, for example $\mathrm{Mo} / \mathrm{MoO}_{-}(3-\mathrm{x})$ for their applications in different types of technologies and magnetoresistance behavior in different types of materials for temperatures close to the critical temperature $\mathrm{T}$ c and more importantly, the study and analogy of the conservation of the spin load in the superconducting state, ie where the spin flows without losses in the superconducting state, behavior that It can be used in systems that emulate the logic gates or spitronic which is one of the branches called to replace the current electronic. Thus, the study of the behavior of the state of vortices in the presence of external current and the dynamics of the vortices given this inclusion of current and the study of depreciation of the superconducting state, due to the presence of an external magnetic field gives an account of possible effects on the state of vortices, which are extremely important in terms of the manipulation of vortices in mesoscopic samples [9-16]. Given this, in this paper we present the behavior of the vortices in a superconducting nano/prism with the inclusion of depreciation of the superconducting state in a given section (deformed system in the Ginzburg-Landau theory), which in our system, establishes the addition of metal contacts, where the inclusion of the current in the system would be carried out, we will study the effect on the magnetization, vortex state and susceptibility, of the external current and temperature of the defects.

\section{THEORETICAL FORMALISM}

We consider an anisotropic rectangle in presence of an external Lorentz force with a central defect of a different critical temperature. The sample is very thin,d $\ll \xi$, where $\xi$ is the coherence length and $\lambda$ is the London penetration depth. Within this approximation, we can neglect the magnetic field produced by the transport current itself. Therefore, it can be treated as a two-dimensional problem The formalism used to study the system considered in Figure 1(a-b) is given by the time-dependent Ginzburg-Landau (TDGL) equations [17-22].

$$
\begin{aligned}
& \frac{\mu}{\sqrt{1+\Gamma^{2}|\psi|^{2}}} \Theta=-(i \nabla-\boldsymbol{A})^{2} \psi+\psi\left(\tau-|\psi|^{2}\right) \\
& \frac{\partial \boldsymbol{A}}{\partial t}=\operatorname{Re}\left[\psi^{*}(i \nabla-\boldsymbol{A}) \psi\right]-\kappa^{2} \nabla \times \nabla \times \boldsymbol{A}
\end{aligned}
$$

In the equations (1) and (2) we have, $\Theta=\left[\partial \psi / \partial t+\Gamma^{\wedge} 2 / 2\right.$ $\left.\left(\partial|\psi|^{\wedge} 2\right) / \partial \mathrm{t}+\mathrm{i} \phi \psi\right], \psi$ is the orden parameter, $\mathrm{A}$ is the potential vector, $\kappa$ is the Ginzburg-Landau Parameter (GLP), $\tau$ is related with the local temperature of the defects, $\phi$ is the scalar potential. We take $\Gamma=10$, $\mu=2.5$, which are taken from the microscopic character of the superconductivity. The equations are presented in an adimensional form, as follows: $\psi$ in units of $\psi \infty$, lengths in units of $\xi$; $\mathrm{A}$ in units of H_c2 $\xi$, where $H \_c 2$ is the second critical field, T the temperature in units of the critical temperature T_c, time in units of Ginzburg-Landau time t_GL $=\pi \hbar 8 \mathrm{k} \_\mathrm{B}$ T_c, scalar potential $\Phi$ in $\phi \_0=\hbar\left(2 \mathrm{et} \_\mathrm{GL}\right)$, the external applied current $\mathbf{J}$ in units of $J_{-} 0=c \sigma \hbar(2$ et GL ), where $\sigma$ is the conductivity in the normal state. We use $\mathrm{T}=0$ for all simulations. The size of the grid is $\delta_{-} \mathrm{x}=\delta_{-} \mathrm{y}=0.1 \xi$. The usual boundary condition is:

$\mathrm{n} \cdot(\mathrm{i} \nabla-\mathrm{A}) \psi=0, \quad$ out of the contacts

$\mathrm{n} \cdot(\mathrm{i} \nabla-\mathrm{A}) \psi=\gamma \psi=\gamma(1-\delta / \mathrm{b}) \psi$ in the contacts

The equations (3) and (4) represents the ususal superconductingany material interface and $b$ is the deGennes parameter. Thus $\gamma=0$ simulates an interface at the normal state, $0<\gamma<1$ simulates a superconductor-metal interface $(\mathrm{b}>\delta) ; \gamma=1$, simulates a superconductor vacuum interface $(\mathrm{b} \rightarrow \infty)$; and a superconductorsuperconductor interface is described by $\gamma>1(b<0)$. Our study is organized as follows: Case (a) we considered the system showed in the Figure 1 (a) taking $\mathrm{J}=0.5, \tau=0$, for several values of the magnetic field $\mathrm{H}$ and $\gamma$, analizying its influence of magnetization $-4 \pi \mathrm{M}$, magnetic susceptibility $\chi \_m$ and superconducting electronic density $|\psi|^{\wedge} 2$. Case (b) we considered the system showed in the Figure 1(b) for several values of $\gamma$ and $\tau$ remain the magnetic field and applied current constant. $\tau(\mathrm{x}, \mathrm{y})$ is a local critical temperature function; $\tau<1(\tau>1)$ for a defect of lower (greater) critical temperature. To calculate the magnetic susceptibility, we used:

$$
\begin{aligned}
& \chi_{m}=\frac{\partial M}{\partial H} \\
& 4 \pi \boldsymbol{M}=\langle\boldsymbol{H}\rangle-\boldsymbol{H}
\end{aligned}
$$

In the equations (5) and (6), $\mathrm{M}$ is the magnetization and $\langle\mathrm{H}\rangle$ is te mean value of the magnetic induction. The equations (5) is numerically solved using the Runge-Kutta method of fifth order.

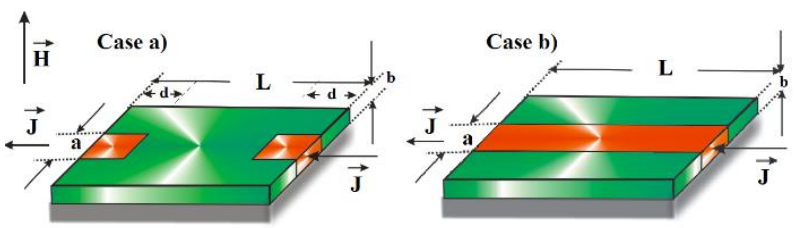

Figure 1. Layout of the studied cases, superconducting square nanoprism of areas: $A=10 \xi \times 8 \xi$ for the case a) and $A=24 \xi \times 12 \xi$, for case $b$ ). $a=2 \xi$, $\mathrm{b}=0.5, \mathrm{~d}=0.1 \xi$, and $\mathrm{J}=0.5$. L is the longest size.

Source: Own elaboration

\section{RESULTS}

Since the magnetization curve accounts for the entry or exit of fluixoids in the simple, but not for its possible movement in the sample, the weak Lorentz force $F_{-} L=J \times H$, does not appreciably change the vortex movement in the dynamics, but is strong enough to influence the vortex matter in the sample, simulating a vortex river guide.

In the Figure 1, we show the layout $\mathrm{f}$ the studies cases. $\tau(\mathrm{x}, \mathrm{y})$ is presented in the superior red zones and $\gamma$ vary in the contacts (lateral red zones), for rest of the sample $\tau(\mathrm{x}, \mathrm{y})=1.0$, (green zones). In the Figure 2(a,b,c). we plot the magnetization as a magnetic field function for the case (a), for a) the contacts are made of another superconducting material at higher critical temperature, b,c) the contacts are made of a metallic material, for $J=0.5,1.2$. $H \_1$ is the magnetic field for the first vortex entry in the sample. We can see that for $\gamma=0.8,0.9$ for $\mathrm{H}>0.75$ the sample remains in the Meissner state, also H_1 is independent of $\gamma<1.1$ (Figure 2(a,b)). However, the magnetization curves present a non conventional behaviour, the Meissner state (Linear parte of the curve) reamains ever after reaching H_1. This behavior that accounts for possible oscillations of the vortices around their equilibrium position before the entry of additional vortices in the sample. 
In the Figure 3 and Figure 4, we present the magnetic susceptibility $\chi_{\_} \mathrm{m}$ for several $\gamma$ parameters. As is well know, when the $\mathrm{H}$ is increased, at a certain point H_1 a Meissner- Shubnikov state transition occurs. At this point, the susceptibility, magnetization and number of vórtices, jumps abruptly, if the magnetic field is increased, the magnetic susceptibility, and magnetization, decreases until another vortex penetrates when it jumps occur, the vorticity $\mathrm{N}$ always invcreases. This continues until the superconducting-normal state transitions. We can see that in the Figures 3(b-d) there is no appreciable variation of $\mathrm{H}_{-}$1. While in Figure 3(a) this variation is observed, being a result compatible with Figures 2. It can be seen in Figures 3, that for $\mathrm{H}>1.2$, the susceptibility is null in all cases. We can see that for the susceptibility there is a behavior that is not as smooth, this is due to a possible additional vortex vibration in their equilibrium position, due to the external current. Already in Figure 4(a), it can be seen that $\chi_{\_} \mathrm{m}$ is canceled for smaller values of the magnetic field, this is the result of the nature of the contact material $(\gamma<1$ (metallic contact), $\gamma<1$ (superconductig contact). So, the system has a more efficient response, if the contacts are made of another superconducting material at higher critical temperature. On the another hand, the discontinuities in the magnetic susceptibility is less abrupt when we take a superconducting contact. This behavior is due to proximity effects between two materials in different phases. When the metal and superconducting at higher critical temperature internal boundary conditions are used in the contacts, we found that the highest values of $\chi \_m$ are rindependent of $\gamma$, so, our results show that the highest (and lowest) values of magnetic susceptibility are independent of the temerature of the defects.
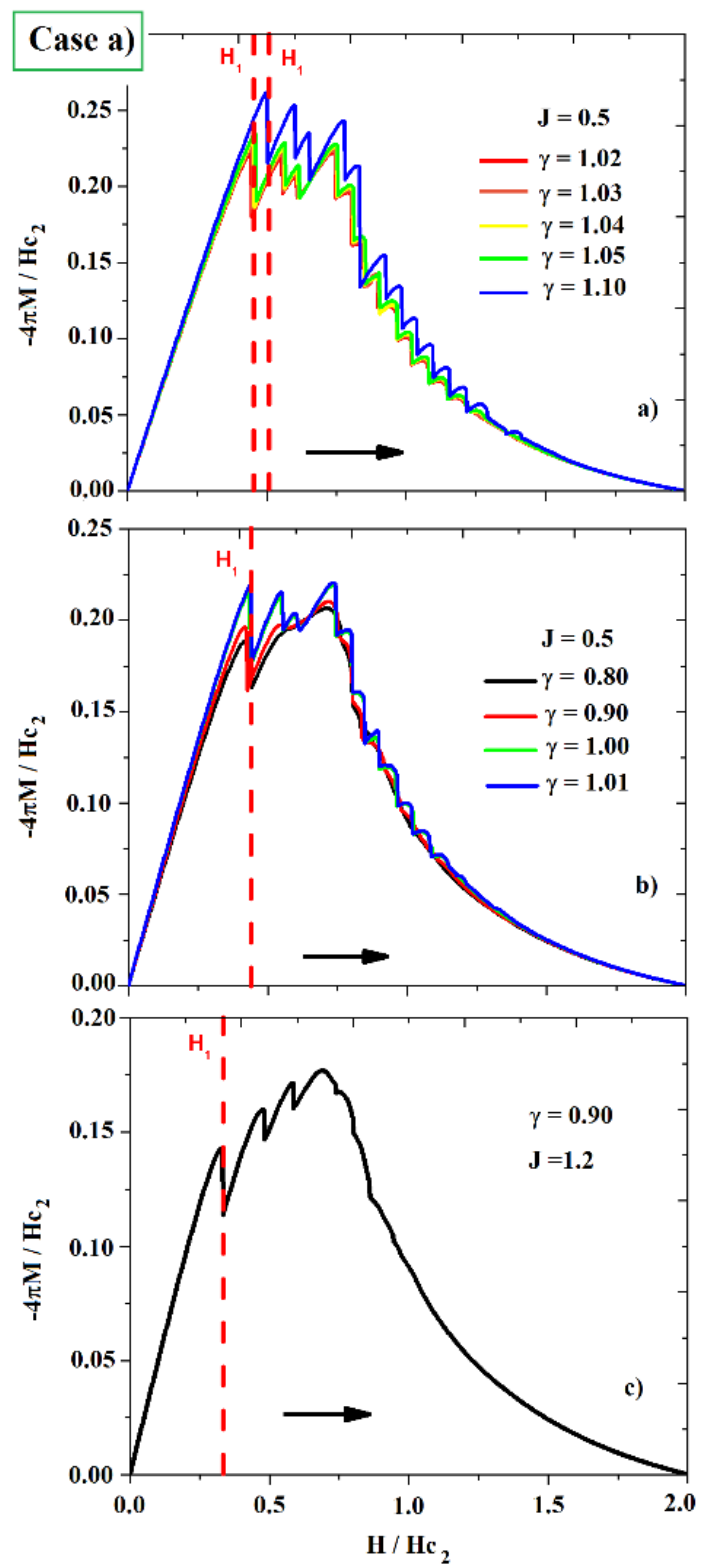

Figure 2. Magnetization curves as a magnetic field function for the case a), for several values of $\boldsymbol{J}$ and $\gamma . H_{1}$ is the magnetic field for which the first vortex entry in the simple.

Source: Own elaboration.

In the Figure 5 (case a) and Figure 6 (case b), we plot the vortex configuration in stationary states at indicates magnetic fields. It is observed how is the entry and configuration of the vortices in the superconducting material and how is the movement of these vortices in the sample. In the case for $\gamma=0.8$, we can see that the entry of the vortices in the sections outside the contacts is slower than in the case presented for $\gamma=1.1$, establishing that for the superconducting system, the superficial energy barrier is lower when we take $\gamma>1$.

Lets now to analyze the case b). In this configuration, the defect present in the sample crosses the superconducting rectangle. Initially we present the effect that magnetization has on the value change for $\tau$ and subsequently on $\gamma$ in the sample. In the Figure 6, the vortex penetrates by the contacts, invreasing the magnetic field, they move perpendicularly to the applied corrent until the up to half of the sample, where they repel parallely to the Lorentz force. The applied current is under the critical current, is very weak, and the movement of the vortex, always obeys the force due to magnetic pressure. When $\gamma>1$ valus in taken less vortices remain in the superconducting region. Still concerning Figure 5 and Figure 6, similar behavior occurs for both studied cases, but with a subtle difference. The vortex configuration at the center of the square is symmetrical in the case b) and assymetrical 
in the case a). Indeed, $\psi$ is minimal at the center of the defect. Notice that the vortices move away from each other symmetrically (assymetrically) with respect to the center of the square and, $\psi$ nearly vanishes across the central channel. This behavior is due to the pinning center force of the vortex guide.
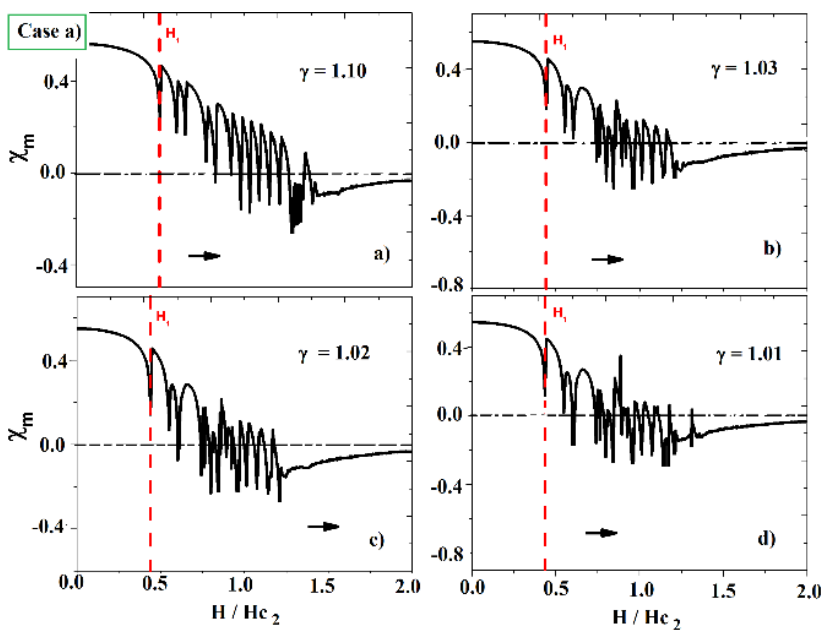

Figure 3. Magnetic susceptibility as a magnetic field function for the case a), for $J=0.5$ and several values of $\gamma>1$.

Source: Own elaboration.

In the Figure 7 and Figure 8, we plot the magnetization and vortex number, as a magnetic field function for the case b) for several $\gamma$ and $\tau$ values. We can appreciate that $H_{-} 1$ depends strongly on $\tau$, is a results evident, due that the Beam-Levingston energy barrier dimished when the temperature and the degennes parameter, so, $\mathrm{H} \_1$ invreases when $\gamma$ and $\tau$ increases. Thus the differents $H_{-} \_$is due the temperature of the defects and nature of the contacts, as is well know, as $\gamma \rightarrow 0$, the Beam Livingston barried energy diminished and , $\mathrm{H}_{-} 1 \rightarrow 0$. Nevertheless, in the Meissner phase, which is common to all the cases, the overall value of the maximun is less when a metalic contacs is used. At high magnetic fields ( $\mathrm{H} \geq 1.0$ ), in the Figure $8(\mathrm{~b}, \mathrm{c})$, the $\mathrm{N}(\mathrm{H})$ curve show a soft vortex transitions, demonstrating the presence of a high magnetic quantized flux into the simple, this fact, can difficult the typical abrut vortex transitions. The magnetic field penetrates the simple continously until reaching $\mathrm{H} \_2$ (superconducting normal transition magnetic field).

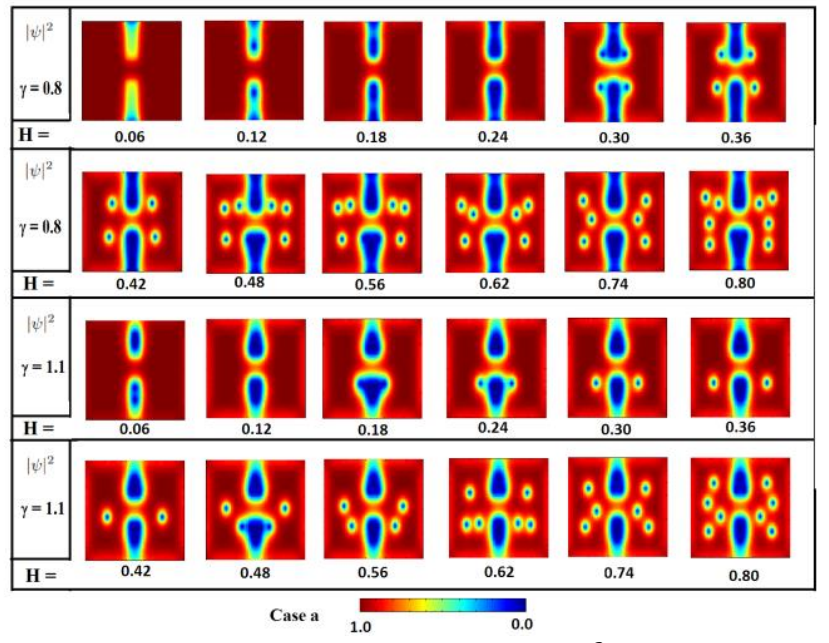

Figure 5. Superconducting electronic density $|\psi|^{2}$ for several magnetic fields for the case a), for $J=0.5$

Source: Own elaboration.

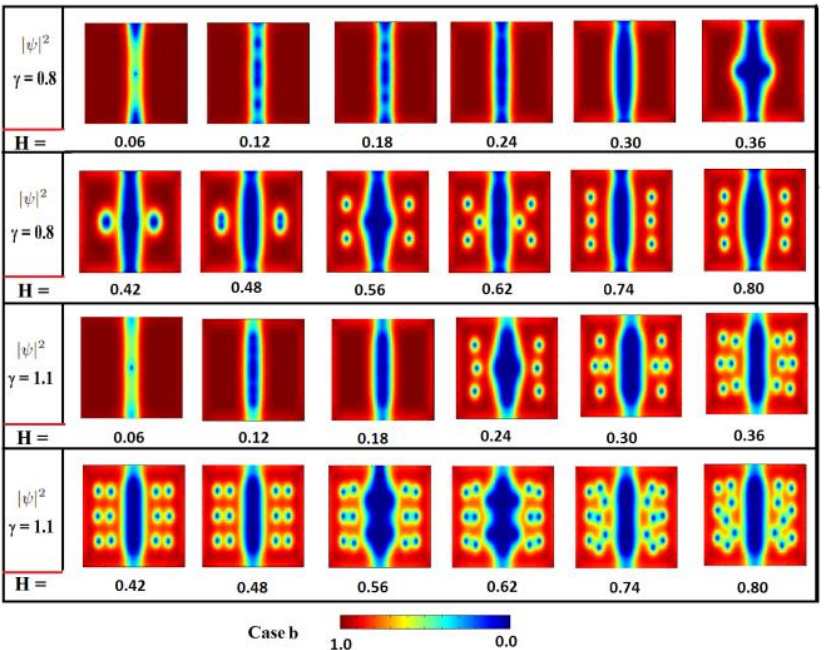

Figure 6. Superconducting electronic density $|\psi|^{2}$ for several magnetic fields for the case $\mathrm{b}$ ), for $J=0.5$.

Source: Own elaboration.


Figure 7. (a) Magnetization and (b) vortex number $\mathrm{N}$, as a magnetic field function for the case $\mathrm{b}$ ), for $J=0.5, \tau=0$, for the indicated $\gamma$ values.

Source: Own elaboration.

The growth of H_1 means that the higher the $\gamma$ factor is, more diamagnetic the material is, as is well known from an experimental point of view, since the shielding of the first penetration of vortices could be enhanced by the inclusion of defects made from another superconductor with a higher critical temperature [23]. Now for the vorticity $\mathrm{N}(\mathrm{H})$, the continuous vortex entry of the first $\mathrm{N}=4$ vortices can be seen for metallic contacts, and $\mathrm{N}=6$ vortices can be seen for a superconducting contact. 

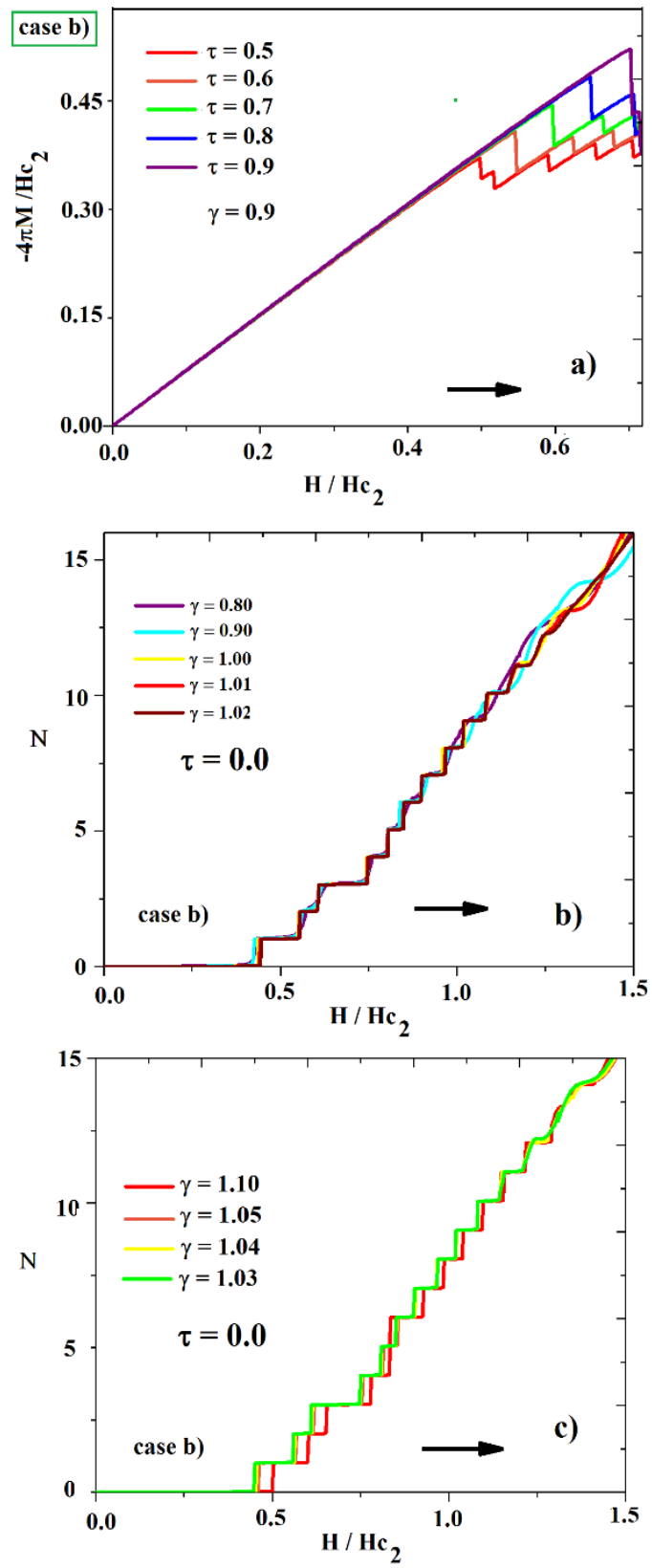

Figure 8. (a) Magnetization and (b,c) vortex number N, as a magnetic field function for the indicated values.

Source: Own elaboration.

\section{CONCLUSIONS}

By solving the time-dependent Ginzburg- Landau equation, we analyzed the superconducting state of a rectangle with topological defects with different critical temperatures under a weak applied Lorentz force. We studied the effect of the temperatura of the defects and the boundary conditions of the contacts on the magnetic susceptibility, magnetization, vorticyty and Cooper pairs. We found that the critical field for the transition to the normal state $\mathrm{H}_{-} 2$, 『( $(\mathrm{H})$

_1) is indepedent (depend strongly) of the geometry, temperature of the defects, and on the boundary conditions used in the contacts where the extrernal current is applied. We found that for a small defect, at high magnetic fields, the vortex configuration is non-symmetrically due to the asymmetry of the supercurrent in the sample. Thus our results are of experimental importance, since the vortices can be controlled by including defects of other superconducting materials with different critical temperatures [24].

\section{REFERENCIAS}

[1] J. Barba-Ortega, E. Sardella y J. Albino-Aguiar, "Superconducting boundary conditions for mesoscopic circular samples, Supercond. Sci. Technol. 24, 015001, 2011.

[2] C. Aguirre, M.R. Joya, J. Barba-Ortega, "Effect of anti-dots on the magnetic suscepti- bility in a superconducting long prisma", J. Low Temp. Phys. 186, 250, 2017.

[3] P.G. de Gennes y J. Matricon, "Collective modes of vortex lines in superconductors of the second kind", Rev. Mod. Phys. 36, 45, 1964.

[4] L. Komendova, M.V. Miloševic, A.A. Shanenko y F.M. Peeters, "Different length scales for order parameters in two-gap superconductors: extended Ginzburg-Landau theory", Phys. Rev. B 84, 064522, 2011.

[5] N.V. Orlova, A.A. Shanenko, M.V. Miloševic', F.M. Peeters, A.V. Vagov y V.M. Axt, "Ginzburg-Landau theory for multiband superconductors: microscopic derivation", Phys. Rev. B 87, 134510, 2013.

[6] B. Xu, M.V. Miloševic y F.M. Peeters, "Calorimetric properties of mesoscopic super- conducting disks, rings, and cylinders", Phys. Rev. B 81, 064501, 2010.

[7] A.V. Silhanek, L. Van Look, S. Raedts, R. Jonckheere y V.V. Moshchalkov, "Guided vortex motion in superconductors with a square antidot array", Phys. Rev. B 68 (2008).

[8] A. Van-Blaanderen, R. Ruel y P. Wiltzius, "Template directed colloidal crystallization", Nature, 385, 321, 1997.

[9] J. Barba, E. Sardella y R. Zadorosny, "Influence of the deGennes extrapolation parameter on the resistive state of a superconducting strip", Phys. Lett. A 382, 215, 2018.

[10] I.N. Askerzade, "Numerical simulation of vortex nucleation in the two-band Ginzburg-Landau model", Tech, Phys., 55, 896, 2010.

[11] X. Wang, S.R. Ghorbani, S.I. Lee, S.X. Dou, C.T. Lin, T.H. Johansen, K .H. Müller, Z. X. Cheng, G. Peleckis, M. Shabazi, A.J. Qviller, V. . Yurchenko, G.L. Sun y D.L. Sun, "Very strong intrinsic flux pinning and vortex avalanches in $(\mathrm{Ba}, \mathrm{K}) \mathrm{Fe}_{2} \mathrm{As}_{2}$ superconducting single crystals", Phys. Rev. C 82 (2010) 024525.

[12] T. Golod, A. Rydh, y V.M. Krasnov, "Detection of the phase shift from a single Abrikosov vortex", Phys. Rev. Lett. 104, 227003, 2010.

[13] E.D. Gulian, G.G. Melkonyan y A.M. Gulian, "Directed motion of vortices and annihi- lation of vortex/antivortex pairs in finite gap superconductors via hot lattice", Phys. Lett. A 381, 2181, 2017.

[14] D. Castelvecchi, "Quantum computers ready to leap out of the lab in", Nature 541 9, 2017.

[15] R. Zadorosny, E.C.S. Duarte, E. Sardella, W.A. Ortiz, "Vortex/antivortex annihilation in mesoscopic superconductors with a central pinning center", Physica, C 503, 94, 2014.

[16] G.R. Berdiyorov, A.D. Hernández-Nieves, M.V. Miloševic', F.M. Peeters y D. Dominguez, "Flux-quantum-discretized dynamics of magnetic flux entry, exit, and annihilation in currentdriven mesoscopic Type-I superconductor", Phys. Rev. B 85, 092502, 2012.

[17] Q. Du, "Numerical approximations of the Ginzburg-Landau models for superconductivity", J. Math. Phys. 46, 095109, 2005.

[18] M.P. Sørensen, N.F. Pedersen y M. Ögren, "The dynamics of magnetic vortices in type II superconductors with pinning sites studied by the time dependent Ginzburg- Landau model", Physica C 533, 40, 2017.

[19] R. Wördenweber, E. Hollmann, J. Schubert, R. Kutzner y A.K. Ghosh, "Vortex motion in high Tc films and a micropattern induced phase transition", Physica C 470, 835-839, 2010. 
[20] C. Aguirre, H. Blas y J. Barba-Ortega, "Mesoscale vortex pinning landscapes in a two component superconductor", Physica C 558, 8, 2018.

[21] C.A. Aguirre, Q. Martins y J. Barba-Ortega, "Analytical development of Ginzburg- Landau equations for superconducting thin film in presence of currents", Rev. UIS Ing. 18 (2), 213, 2013.

[22] C.A. Aguirre, M.R. Joya y J. Barba-Ortega, "Dados como centro de anclaje topológico en una muestra superconductora, Rev. UIS Ing. 19 (1), 109, 2020.

[23] L. Salasnich, A.A. Shanenko, A. Vagov, J. Albino Aguiar y A. Perali, "Screening of pair fluctuations in superconductors with coupled shallow and deep bands: A route to higher-temperature superconductivity", Phys. Rev. B 100, 064510, 2019.

[24] E. Marchiori, J, Peter-Curran, K. Jangyong, N. Satchell, G. Burnell y S.J. Bending, "Reconfigurable superconducting vortex pinning potential for magnetic disks in hybrid structures", Scientific Reports, 7 (2017) 45182. 